\title{
脱氢偶联制备芳基醚方法的研究进展
}

\author{
唐 影 ${ }^{a}$ 骆钧飞*,a 解 攀*,b \\ ( ${ }^{a}$ 宁波大学材料科学与化学工程学院 宁波 315211) \\ ${ }^{b}$ 陕西科技大学化学与化工学院 陕西省轻化工助剂重点实验室 西安 710021)
}

\begin{abstract}
摘要 芳香梄结构是许多天然产物以及药物分子的重要骨架，同时也是非常重要的有机合成中间体. 通常, 芳香醚由 含离去官能团的芳烃与醇或酚偶联制得. 此类方法由于需对底物进行预官能团化, 因此存在着低原子及步骤经济性不 足等问题. 近年来, 利用过渡金属催化的 $\mathrm{C}-\mathrm{H}$ 键活化策略，通过芳烃与醇或酚的脱氢偶联实现芳基醚的制备，越来越 受到有机化学家关注. 综述了最近通过脱氢偶联制备芳香醚化合物的研究进展. 围绕各类方法的底物适应范围和反应 机理等进行详细论述, 并就该领域的局限性和未来的发展前景进行总结和展望.
\end{abstract}

关键词 芳香醚; 脱氢偶联; $\mathrm{C}-\mathrm{H}$ 键活化; 苯酚; 醇

\section{Advances on the Synthesis of Aryl Ethers via Dehydrogenative Coupling}

\author{
Tang, $\mathrm{Hao}^{a} \quad$ Luo, Junfei ${ }^{*, a} \quad \mathrm{Xie}, \mathrm{Pan}^{*, b}$ \\ ( ${ }^{a}$ School of Materials Science and Chemical Engineering, Ningbo University, Ningbo 315211) \\ ( ${ }^{b}$ Shaanxi Key Laboratory of Chemistry Additives for Industry, College of Chemistry and Chemistry Engineering, \\ Shaanxi University of Science \& Technology, Xi'an 710021)
}

\begin{abstract}
Aryl ethers are important central motifs that are abundant in many natural products and drug molecules, as well as versatile building blocks for organic synthesis. Aryl ethers were usually synthesized through the coupling reactions between leaving group substituted arenes and alcohols. However, the introduction of leaving group requires extra synthetic operation and produces lots of wastes. Over the past decade, the method for the synthesis of aryl ethers via $\mathrm{C}-\mathrm{H}$ alkoxylation or aryloxylation has received much attention due to its potential as an atom and step efficient methodology. Herein, the research advances on the synthesis of aryl ethers through dehydrogenative coupling are reviewed. The detailed substrate scopes and reaction mechanisms, as well as the limitations of current procedures and the prospects for the future, are discussed.

Keywords aryl ether; dehydrogenative coupling; $\mathrm{C}-\mathrm{H}$ activation; phenol; alcohol
\end{abstract}

芳香醚骨架广泛存在于天然产物和药物分子中, 比 如 BMS-777607 (muti-kinase inhibitor, 多激醚抑制剂)、 antitumor riccardin C(riccardin C 靶向抗癌药)、antithyroid levothyroxine(抗甲状腺左甲状腺素)、Gefitinib (EGFR inhibitor, EGFR 抑制剂)以及 Vitamin $\mathrm{E}$ (维生素 $\mathrm{E}$ )(图 $1)^{[1]}$. 另外, 芳基醚还是重要的有机合成中间体, 通过 切断芳基 $\mathrm{C}-\mathrm{O}$ 键, 可以引入不同的官能团, 从而实现 不同功能有机化合物分子的合成 ${ }^{[2]}$. 传统的芳香醚类化 合物的合成是通过 Ullmann 反应(Scheme 1, a) 实现的,
但是该反应需要等物质的量或过量的铜作为催化剂, 且 需要较高的反应温度 $\left(200 \sim 350{ }^{\circ} \mathrm{C}\right)^{[3]}$. Chan-Evans-Lam 反应改进了 Ullmann 反应，它使用芳基硼酸代替卤代物, 利用催化剂量的铜即可实现芳香醚化合物的制备 ${ }^{[4]}$. 相 对而言, Buchwald-Hartwig 交叉偶联反应提供了更为有 效的策略，其通过钯催化的芳基卤代烃与醇或酚的偶联 实现芳香醚化合物的制备 (Scheme 1, b $)^{[5]}$. 最近, MacMillan 利用光催化氧化还原的方法, 发展了镍和铱的双 金属催化策略，实现了芳基卤代烃与醇的偶联反应 ${ }^{[6]}$.

\footnotetext{
* Corresponding authors. E-mail: luojunfei@nbu.edu.cn, pan.xie@sust.edu.cn Received April 4, 2019; revised May 9, 2019; published online June 6, 2019. Project supported by the Natural Science Foundation of Zhejiang Province (No. LQ19B020002), the Municipal Natural Science Foundation of Ningbo City (No. 2018A610241), the Education Foundation of Zhejiang Province (No. Y201839228) and the K. C. Wong Magna Fund in Ningbo University. 浙江省自然科学基金(No. LQ19B020002)、宁波市自然科学基金(No. 2018A610241)、浙江省教育厅(No. Y201839228)和宁波大学王宽诚幸福基金资助 项目.
} 


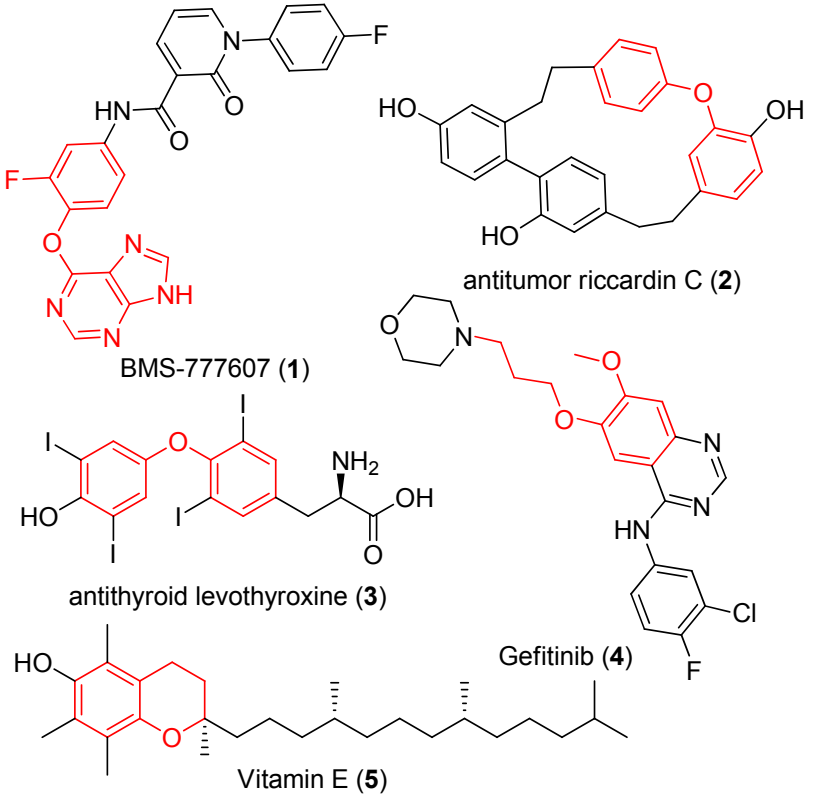

图 1 含芳香醚结构的重要分子

Figure 1 Important molecules containing aryl ethers

(a) Ullmann

$\mathrm{Ar}-\mathrm{X}+\mathrm{HO}-\mathrm{R} \stackrel{[\mathrm{Cu}]}{\longrightarrow} \mathrm{Ar}-\mathrm{O}-\mathrm{R}+\mathrm{HX}$

(b) Buchwald-Hartwig corss-coupling

$$
\mathrm{Ar}-\mathrm{X}+\mathrm{H}_{2} \mathrm{O}-\mathrm{R} \stackrel{[\mathrm{Pd}]}{\longrightarrow} \mathrm{Ar}-\mathrm{O}-\mathrm{R}+\mathrm{HX}
$$

(c) Synthesis of aryl ethers via $\mathrm{C}-\mathrm{H}$ activation

$$
\mathrm{Ar}-\mathrm{H}+\mathrm{HO}-\mathrm{R} \stackrel{[\mathrm{TM}]}{\longrightarrow} \mathrm{Ar}-\mathrm{O}-\mathrm{R}+\mathrm{H}_{2}
$$

图式 1 芳基醚制备方法

Scheme 1 Methods for the preparation of aryl ethers

上述方法中均以含离去基团的芳香烃(芳基卤代烃 或金属试剂)作为反应底物, 其需要对芳香烃进行预官 能团化，因此大大降低了芳基醚的合成效率; 另一方面， 离去基团的引入导致了副产物的增加, 且大多数情况下 会生成毒性较大的含卤副产物或金属废弃物. $\mathrm{C}-\mathrm{H}$ 键 的直接官能化, 即用芳香烃直接与醇或酚偶联, 提供了 另一种更为高效绿色的芳香醚合成方法. 该方法无需预 官能团化引入离去基团, 具有步骤经济性; 且理论上副 产物只有氢气，因此具有较强的原子经济性(Scheme 1, c). 近年来尽管有不少关于 $\mathrm{C}-\mathrm{H}$ 活化 $/ \mathrm{C}-\mathrm{O}$ 键偶联方法 的进展报道 ${ }^{[7]}$, 但基于脱氢偶联制备芳香醚的方法尚未 报道. 本综述对近几年报道的利用 $\mathrm{C}-\mathrm{H}$ 活化策略合成 芳香醚的方法进行了总结, 相信这对芳基醚类化合物绿 色高效合成方法的发展具有一定的帮助.

\section{1 芳香烃和醇脱氢偶联}

\section{$1.1 \mathrm{Pd}$ 催化芳香烃和醇交叉偶联反应}

目前, 钯金属催化惰性键活化是发展最为成熟的
$\mathrm{C}$ - $\mathrm{H}$ 键官能团化方法. 为了提高反应的选择性, 往往 需要引入一个导向基团, 其配位基团可与钯催化剂络 合，诱导 $\mathrm{Pd}$ 催化剂活化其邻位的 $\mathrm{C}-\mathrm{H}$ 键形成环钯中间 体(通常为五元或六元环中间体), 环钯化合物经氧化加 成、还原消除可得到官能团化产物(Scheme 2).

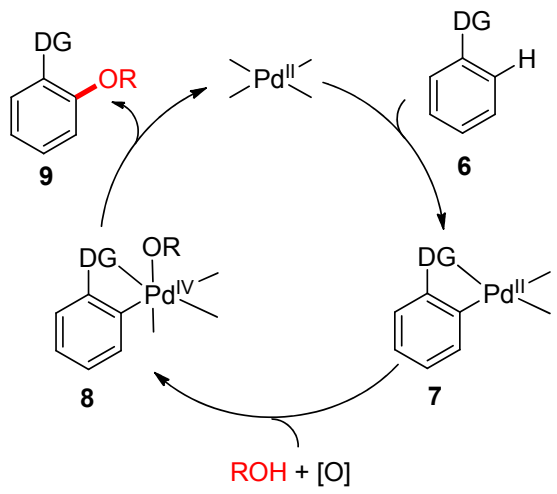

图式 2 Pd 催化 C- $\mathrm{H}$ 活化制备芳基醚机理

Scheme 2 Mechanism for the synthesis of aryl ethers via Pdcatalyzed $\mathrm{C}-\mathrm{H}$ activation

2006, Sanford 课题组 ${ }^{[8]}$ 首次应用了该策略，以肜醚 或吡咯烷酮作为导向基团，在 $\mathrm{Pd}$ 催化下实现了邻位 $\mathrm{C}-\mathrm{H}$ 甲氧基化，完成了茴香醚衍生物的制备(Eqs. 1, 2). 该课题组设计了四价钯金属催化氧化体系，使用廉价、 安全、环保的硫酸氢钾复合物作为氧化剂. 另外甲醇作 为甲氧基源，同时作为反应溶剂，因此提高了反应效率.
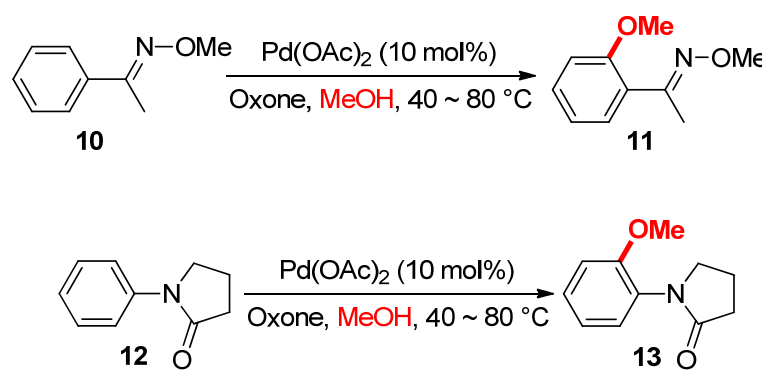

王官武课题组 ${ }^{[9]}$ 以 $N$-甲氧基酰胺基(CONHOMe)作 为导向基团，在类似的反应条件，分别以甲醇、乙醇和 异丙醇作为偶联试剂实现了其邻位 $\mathrm{C}-\mathrm{H}$ 键的烷氧基化 (Eq. 3), 扩大了当前方法的潜在合成应用. 作者在相同 反应条件下尝试了乙酰苯胺邻位 $\mathrm{C}-\mathrm{H}$ 甲氧基化反应, 发现其反应效率大幅降低, 仅以 $30 \%$ 的收率得到官能化 产物. 随后，该课题组 ${ }^{[10]}$ 通过向反应体系中添加催化量 的甲磺酸实现了乙酰苯胺衍生物邻位 $\mathrm{C}-\mathrm{H}$ 键的高效 烷氧基化反应. 反应在室温下即可进行(Eq. 4), 且大量 不同的醇包括甲醇、乙醇、正丁醇、仲丁醇、正丙醇、 异丙醇、2-甲氧基乙醇、2-氯乙醇、环己醇和环戊醇等 均可作为偶联试剂. 作者推测甲磺酸在反应中不仅促进 
了 $\mathrm{C}-\mathrm{H}$ 键的活化，同时也扮演离去基团的角色，从而 提高了反应活性. 在该策略的启发下, 匡春香课题组 ${ }^{[1]}$ 利用相似的反应条件, 以 $1,2,3$-三氮唑作为导向基团实 现了其邻位的 $\mathrm{C}-\mathrm{H}$ 烷氧基化反应.
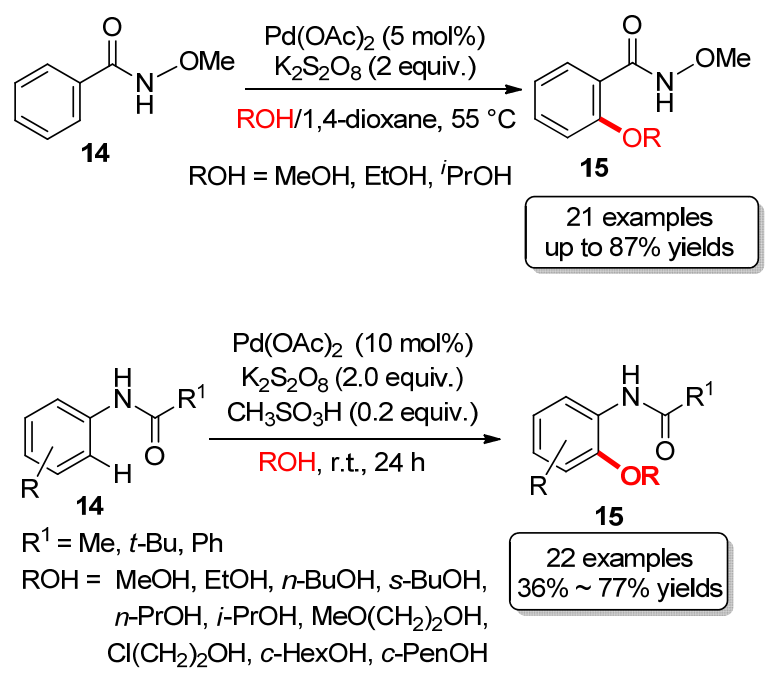

孙培培课题组 ${ }^{[12]}$ 以氰基作为导向基团, 在甲醇或 乙醇存在下, 实现邻位 $\mathrm{C}-\mathrm{H}$ 键的甲氧基化或乙氧基化 (Eq. 5). 当底物无其他取代基或对位取代时反应发生双 $\mathrm{C}-\mathrm{H}$ 官能团化, 而当底物邻位或间位取代时则发生单 $\mathrm{C}-\mathrm{H}$ 官能团化. 与其他导向基团相比, 氮基则可以更 多地衍生化, 比如可还原成胺基或醛基、水解成羧基或 环加成得到四氮唑. 作者认为氰基作为导向基团是通过 $\pi$ 键与钯金属配位形成环钯中间体 19. 乙酰基参与去质 子活化 $\mathrm{C}-\mathrm{H}$ 键, 进一步氧化加成、还原消除得到邻位 甲氧基化产物。

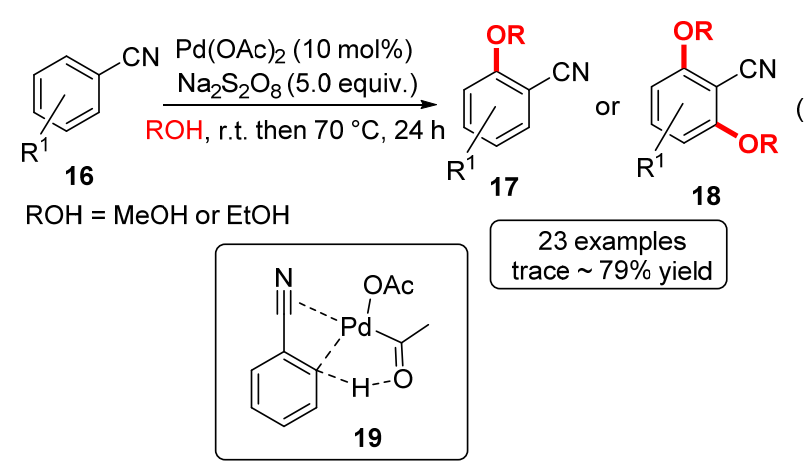

随后，孙培培课题使用偶氮基 ${ }^{[13]} 、 2$-吡啶氧基 ${ }^{[14]}$ 和 $N$-烷基- $N$-亚硝基胺 ${ }^{[15]}$ 为导向基团, 以 $\mathrm{Pd}$ 金属作为催化 剂, 二乙酸碘苯 $\left(\mathrm{PhI}(\mathrm{OAc})_{2}\right)$ 为氧化剂, 烷基醇作为烷氧 化剂和溶剂, 通过邻位 $\mathrm{C}-\mathrm{H}$ 键活化构建芳基醚衍生物 (Eq. 6). 上述的底物均具有较好的官能团耐受性. 底物 $N$-烷基- $N$-亚硝基苯胺可通过铁粉简单处理, 还原生成 苯胺衍生物.

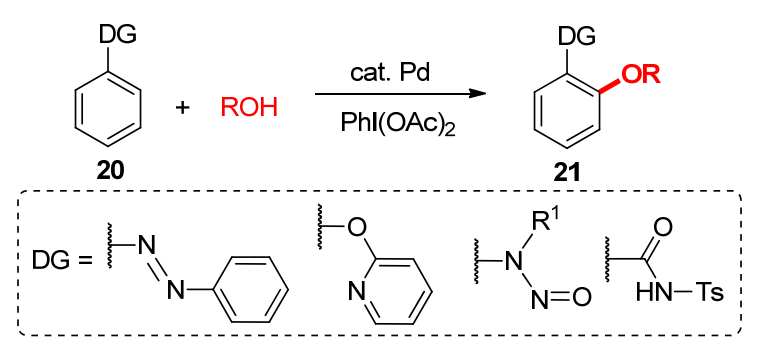

Fabis 课题组 ${ }^{[16]}$ 采用 $N$-甲苯磺酰甲酰胺基团作为导 向基团，在类似的反应条件下，实现邻位 $\mathrm{C}-\mathrm{H}$ 烷氧基 化. $N$-卤代琥珀酰亚胺 (NXS) 代替二乙酸碘苯作为氧化 剂时, 醚化反应并没有顺利进行, 而是在导向基团的邻 位发生了卤代反应。

史炳锋课题组 ${ }^{[17]}$ 发展了新型的双齿导向基团 2-(2吡啶基)-2-胺基丙烷基, 通过其邻位 $\mathrm{C}-\mathrm{H}$ 键的活化实 现苯甲酸衍生物的烷氧基化(Eq. 7). 该导向基团具有易 制备和易脱除等优良特点. 值得注意的是作者利用该导 向基团实现了大量脂肪族羧酸衍生物 $\beta$ 位的 $\mathrm{C}\left(\mathrm{sp}^{3}\right)-\mathrm{H}$ 烷氧基化. 作者通过密度泛函理论(DFT)计算推测 $\mathrm{C}$ $\mathrm{H}$ 键的活化是通过协同金属去质子化过程(CMD) 实现 的.

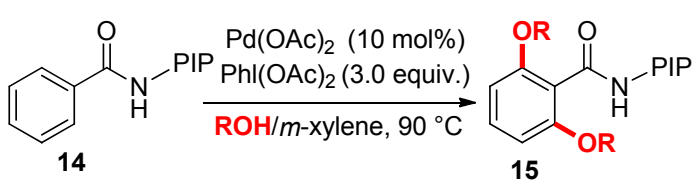

Select examples

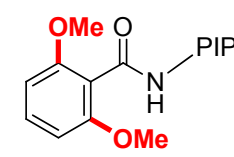

$78 \%$<smiles>Cc1cc(OCC(F)(F)F)c(C(=O)NC(C)C(F)(F)F)c(OCC(F)(F)F)c1</smiles>

\section{$1.2 \mathrm{Pd}$ 催化芳香烃和醇分子内偶联反应}

2,3-二氢苯并呋喃广泛存在天然产物和药物分子 中 $^{[18]}$. 通过芳香烃 $\mathrm{C}-\mathrm{H}$ 活化并与醇羟基发生分子内偶 联, 可快速得到二氢苯并呋喃衍生物. 2010 年, 余金权 课题组 ${ }^{[19]}$ 报道了一种钯催化苯乙醇邻位 $\mathrm{C}-\mathrm{H}$ 键活化进 而实现分子内 $\mathrm{C}-\mathrm{O}$ 键构建的方法(Eq. 8), 该方法可快 速实现二氢苯并呋喃衍生物的制备. 碳酸锂 $\left(\mathrm{Li}_{2} \mathrm{CO}_{3}\right)$ 在 反应中具有重要的作用, 作者认为可能的原因是 $\mathrm{C}-\mathrm{H}$ 键活化的前体钯配合物在非碱性条件下不稳定. 可能的 
催化循环如 Scheme 3 所示: 首先羟基导向 Pd 催化邻位 $\mathrm{C}-\mathrm{H}$ 键活化形成六元环钯中间体 24, 进而氧化剂将其 氧化得到四价钯中间体 25, 最后中间体 25 发生还原消 除反应得到二氢苯并呋喃衍生物, 并重新生成钯催化剂 完成催化循环.
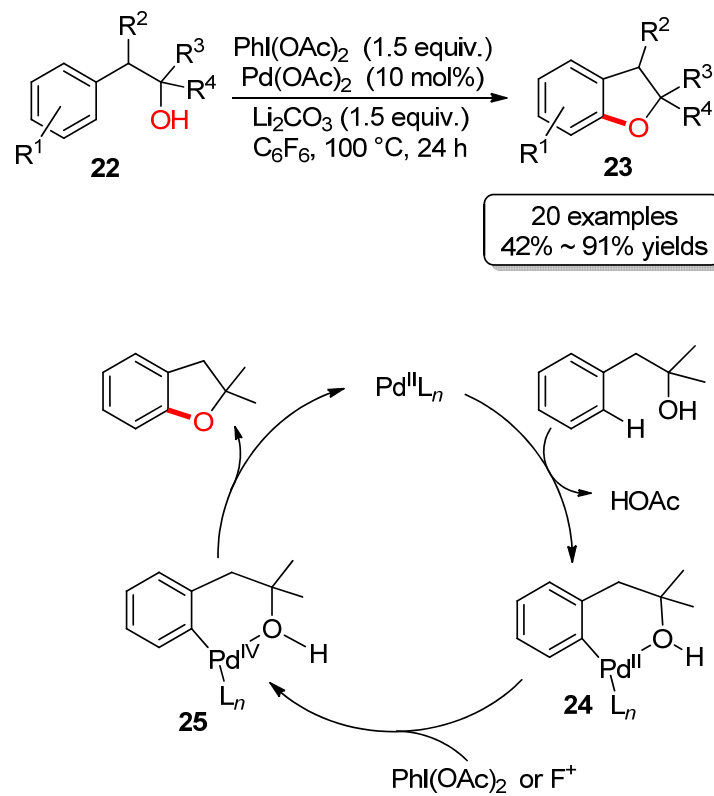

图式 3 钯催化的 $\mathrm{C}-\mathrm{O}$ 环化脱氢机理图 Scheme 3 Pd-catalyzed C-O cyclization

随后，该课题组 ${ }^{[20]}$ 通过连续的 $\mathrm{C}-\mathrm{H}$ 官能团化反应 制备了具有对映选择性的二氢苯并呋喃衍生物. 该方法 首先是叔丁基二甲基甲硅烷基醚(TBS)保护的茮醇在铑 催化下与重氮化合物发生不对称偶联, 生成化合物 26. 酸性条件下化合物 26 中的 TBS 脱保护得到 27 , 其在与 Eq. 8 相同的条件下发生分子内环化得到二氢苯并呋喃 衍生物 28, 产物保留原有的对映选择性(Scheme 4). 产 物 28 中的酯基水解成羧基后还可诱导芳香环上邻位的 $\mathrm{C}-\mathrm{H}$ 烯基化反应. 同年, 余金权课题组 ${ }^{[21]}$ 将该策略拓 展到苯乙酸衍生物的邻位 $\mathrm{C}-\mathrm{H}$ 活化反应, 通过分子内 环化反应实现了苯并呋喃酮的高效合成.

\section{$1.3 \mathrm{Cu}$ 催化芳香烃与醇交叉偶联}

铜作为一种常见的过渡金属，具有良好的兼容性， 且价格相对低廉, 因而受到广泛关注, 其催化脱氢偶联 构建芳基醚的方法亦有不少报道. 王梅祥课题组 ${ }^{[22]}$ 合 成了 Azacalixaromatics 的铜化合物 29, 该化合物在碱 1,8-二氮杂双环 [5.4.0]十一碳-7-烯(DBU)存在下可与醇 或苯酚发生偶联生成芳基醚衍生物(Eq. 9).

2014 年, 宋毛平课题组 ${ }^{[23]}$ 报道了 $N, O$-二齿导向基 团促进的邻位 $\mathrm{C}-\mathrm{H}$ 烷氧基化反应, 需要在等物质的量 的氯化亚铜 $(\mathrm{CuCl})$ 存在的条件下进行 (Eq. 10). 反应具

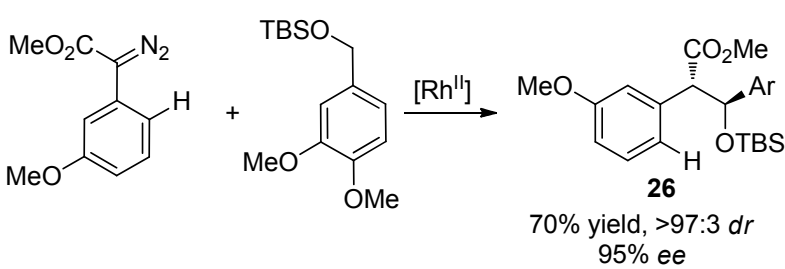<smiles>CCCOc1ccc2c(c1)C(C(C)=O)[C@H]([Al])O2</smiles><smiles>CCOC(=O)/C=C/c1c(OCC(=O)OCC)ccc2c1[C@H](C(=O)O)[C@H](c1ccc(OC)c(OC)c1)O2</smiles>

图式 $4 \mathrm{C}-\mathrm{H}$ 官能化反应合成二氢苯并呋喃衍生物 Scheme 4 C $-\mathrm{H}$ functionalization for the synthesis of dihdrobenzofuran derivatives
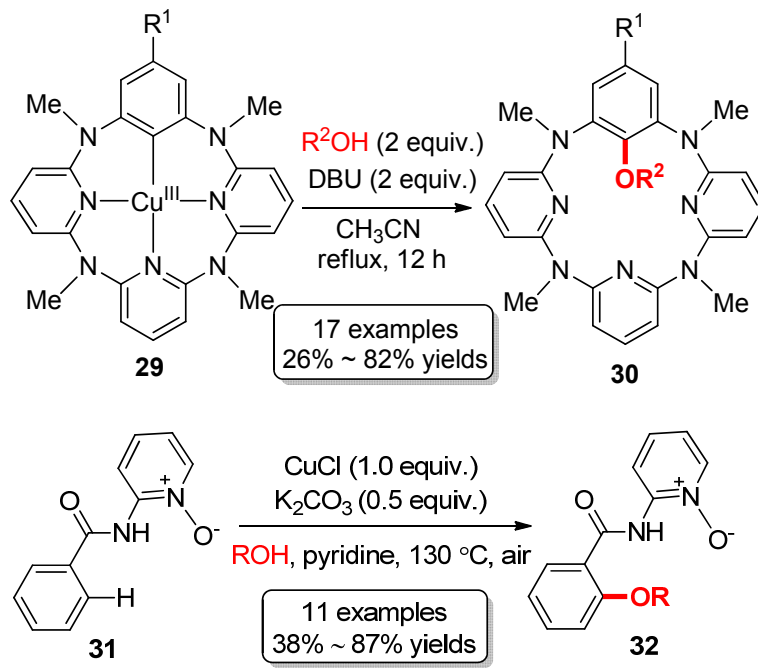

有很好的底物官能团耐受性，卤素、硝基、醚、烷氧基、 酯和磺酰等多种官能团的底物均可顺利发生反应. 作者 推测铜和氧气作用后与醇反应可产生烷氧自由基 $\mathrm{EtO}$ 和超氧二价铜 $\mathrm{ClCuOOH}$. $\mathrm{ClCuOOH}$ 和二齿导向基团生 成二价铜化合物 33, 33 和 $\mathrm{EtO}$ •作用生成三价铜化合物 34, 最后 34 还原消除生成芳基醚产物(Scheme 5).

相比于介导的方法, 金属催化更加绿色环保, 且极 大降低反应成本. 2013 年 Gooßen 课题组 ${ }^{[24]}$ 使用催化量 的醋酸铜 $\left(\mathrm{Cu}(\mathrm{OAc})_{2}\right)$ 实现了含 $\mathrm{N}$ 导向基团的邻位 $\mathrm{C}-\mathrm{H}$ 烷氧基化(Eq. 11). 作者认为可能的反应催化循环如 Scheme 6 所示: 三氟甲磺酸银 (AgOTf)作为醇的活化试 剂, 与醇发生作用生成瞬态烷氧化银 38.38 与环铜化合 物 37 发生单电子转移生成三价铜化合物 39, 并发生还 原消除过程得到芳基醚产物和一价铜 $\mathrm{Cu}(\mathrm{I}) \mathrm{L}_{n}$ ，一价铜 
被氧气氧化重新生成二价铜完成催化循环.

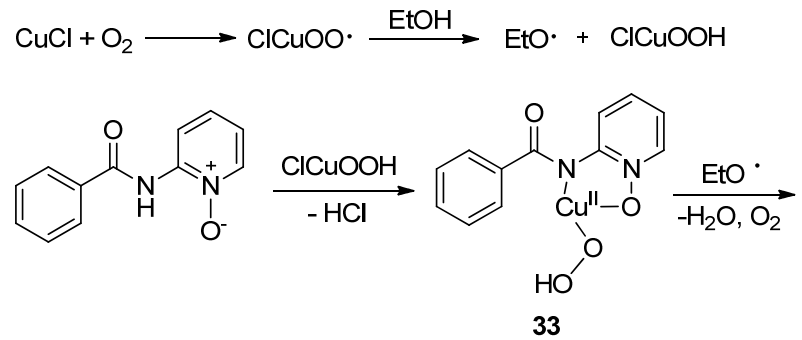

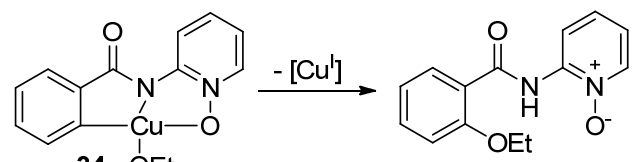

34 OEt

图式 $5 \mathrm{Cu}$ 介导 $\mathrm{C}-\mathrm{H}$ 烷氧基化机理

Scheme 5 Mechanism for $\mathrm{Cu}$-mediated $\mathrm{C}-\mathrm{H}$ alkoxylation
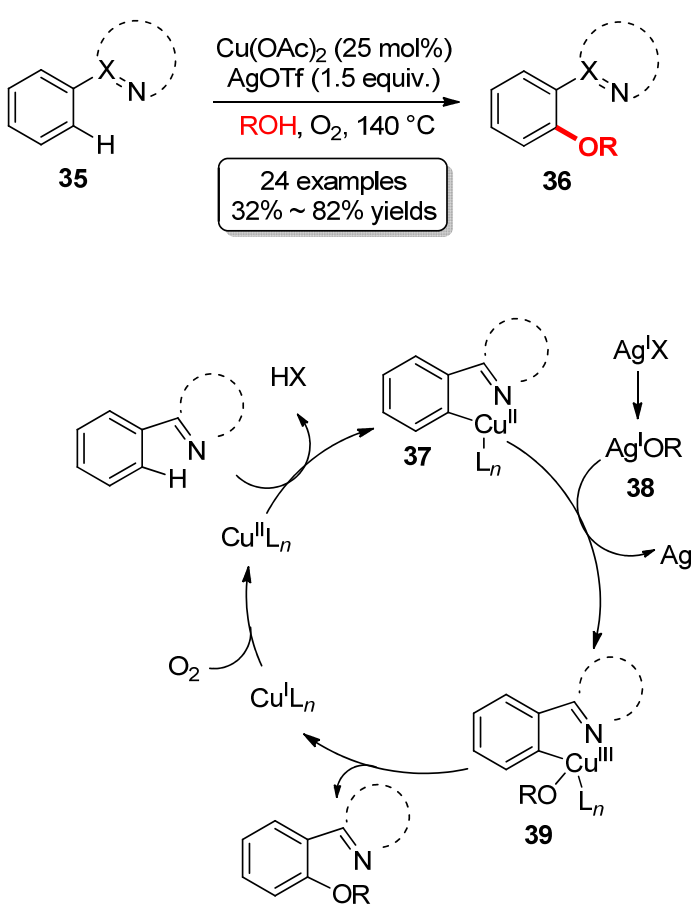

图式 6 铜催化 $\mathrm{C}-\mathrm{H}$ 烷氧基化催化循环图 Scheme 6 Catalytic cycle for $\mathrm{Cu}$-catalyzed $\mathrm{C}-\mathrm{H}$ alkoxylation

同年, Daugulis 等 ${ }^{[25]}$ 以 8-氨基喹啉作为导向基团, 实现了铜催化苯甲酸衍生物邻位的 $\mathrm{C}-\mathrm{H}$ 烷氧基化反应 (Eq. 12). 不同于之前的工作, 该策略以吡啶作为反应溶
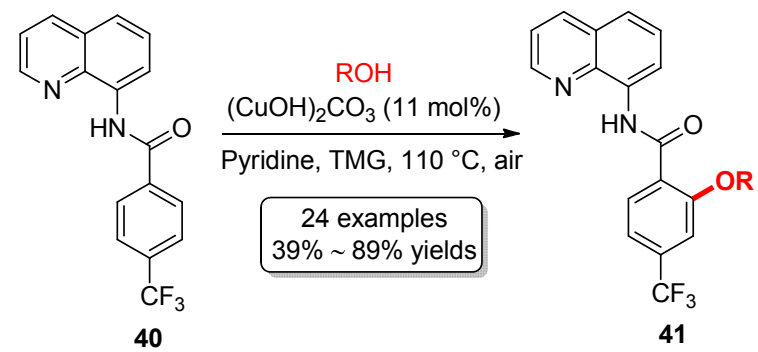

剂，而醇仅仅作为偶联试剂，实现了芳环与多类型醇的 偶联. 但是该反应策略仅对对三氟甲基取代的苯甲酸衍 生物有效。

2015 年，史炳锋课题组 ${ }^{[26]}$ 利用 2-(2-吡啶基)-丙胺 (PIP)作为导向基团，将上述策略拓展应用于更多的芳 香底物(Eq. 13). 反应不仅适用于不同官能团取代的苯 环，同时也适用于杂环化合物，如吡啶、哒嗪和噻吩. 反 应可能的机理如 Scheme 7 所示: 二价铜 $\mathrm{Cu}(\mathrm{II}) \mathrm{X}_{2}$ 首先与 导向基团配位生成二价铜化合物 $\mathbf{4 2}$, 接下来 $\mathbf{4 2}$ 与二价 铜催化剂发生歧化反应生成三价铜化合物 43 和一价铜 $\mathrm{Cu}(\mathrm{I}) \mathrm{X} .43$ 还原消除生成一价铜化合物 44. 最后 44 质子 化得到芳香醚产物和 $\mathrm{Cu}(\mathrm{I}) \mathrm{X} . \mathrm{Cu}(\mathrm{I}) \mathrm{X}$ 被氧化重新生成 $\mathrm{Cu}(\mathrm{II}) \mathrm{X}_{2}$ 催化剂完成催化循环.
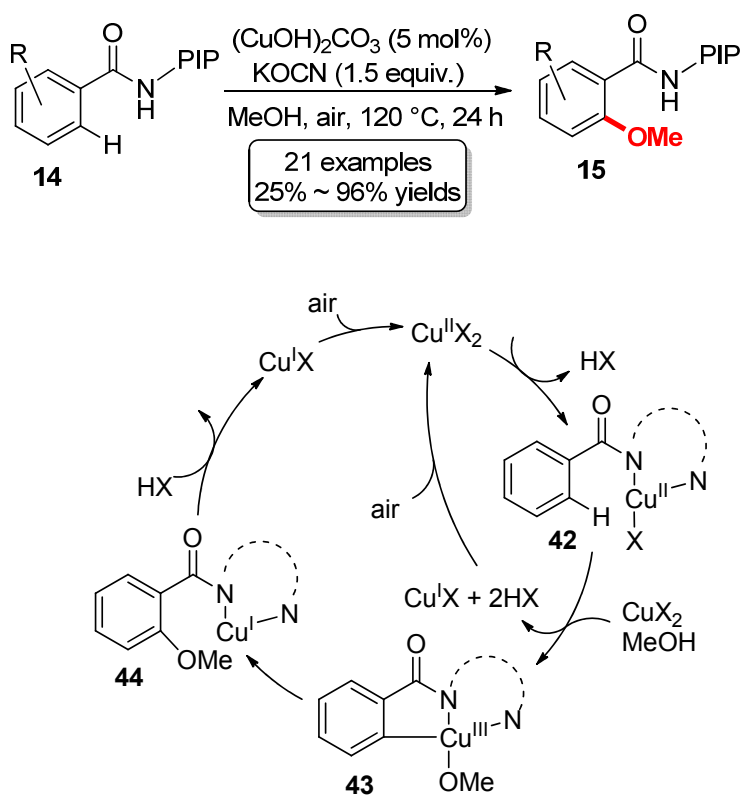

图式 7 一价铜催化 $\mathrm{C}-\mathrm{H}$ 甲氧基化机理图

Scheme 7 Mechanism for $\mathrm{Cu}(\mathrm{I})$-catalyzed $\mathrm{C}-\mathrm{H}$ methoxylation.

\section{4 其他金属催化芳香烃与醇交叉偶联}

2015 年, 宋毛平课题组 ${ }^{[27]}$ 首次报道了钴催化的芳 香烃 $\mathrm{C}-\mathrm{H}$ 键烷氧基化反应(Eq. 14). 作者对不同的导向 基团篮选后发现，2-氨基-吡啶- $N$-氧化物作为双齿导向 基团时，反应效率最高. 尽管反应的机理尚不明确，但 是作者并未发现反应有动力学同位素效应 $\left(\mathrm{KIE}, k_{\mathrm{H}} / k_{\mathrm{D}} \approx\right.$

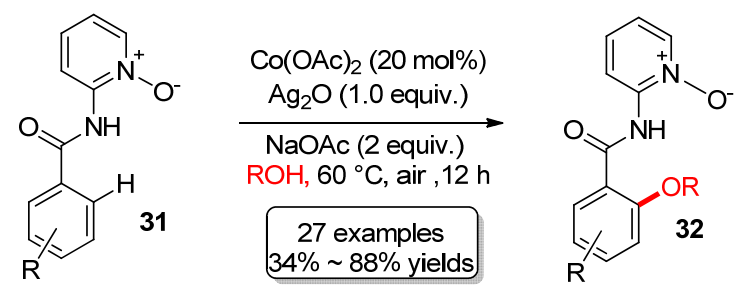


1.0), 表明芳烃的 $\mathrm{C}-\mathrm{H}$ 键断裂不是限速步骤. 另一方 面, 添加自由基捕捉剂 2,2,6,6-四甲基哌啶氮氧化物 (TEMPO)会阻止反应. 因此作者推测该反应可能经历了 自由基过程.

2017 年, 佟振合团队 ${ }^{[28]}$ 发现以醇作溶剂, 苯在光和 钴的协同催化下可以发生脱氢偶联生成芳香醚化合物 (Eq. 15). 该反应在室温下即可发生, 无需额外氧化剂, 氢气为唯一的副产物. 在这之前的报道中反应没有钴催 化剂存在时效率则很低 ${ }^{[29]}$. 反应可能经历的过程如 Scheme 8 所示, 光照下 3-氰基- $N$-甲基喹啉盐 $\left(\mathrm{QuCN}^{+}\right)$ 被激活形成单线态 $\mathrm{QuCN}^{+*}$, 苯环上单电子转移至 $\mathrm{QuCN}^{+*}$ 生成苯环正离子自由基和 $\mathrm{QuCN} \bullet$. 同时, 苯环 正离子自由基与醇在一价钴催化剂 $\mathrm{Co}(\mathrm{I})$ 作用下生成二 烯自由基 47 与三价钴氢化合物 $\mathrm{Co}(\mathrm{III})-\mathrm{H}$. 最后 47 与 $\mathrm{Co}(\mathrm{III})-\mathrm{H}$ 作用脱氢生成芳香醚产物和二价钴 $\mathrm{Co}(\mathrm{II})$. $\mathrm{Co}(\mathrm{II})$ 可与 $\mathrm{QuCN}$ •作用重新生成光催化剂 $\mathrm{QuCN}^{+}$和 Co(I)催化剂完成催化循环.

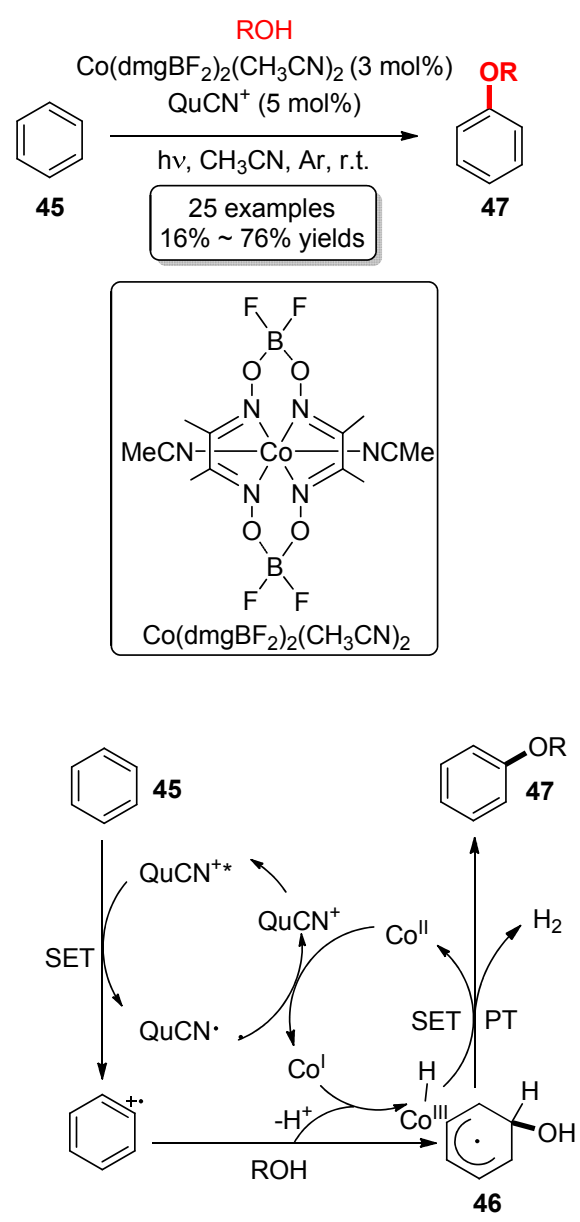

图式 8 钴催化 $\mathrm{C}-\mathrm{H}$ 烷氧基化催化循环图 Scheme 8 Catalytic cycle for Co-catalyzed C-H alkoxylation 2011 年, 赵康和杜云飞等 ${ }^{[30]}$ 发现廉价金属铁也可
促进芳香环 $\mathrm{C}-\mathrm{H}$ 键的醚化反应(Eq. 16). 该方法可快速 制备一系列的香豆素类似物. 氯化铁 $\left(\mathrm{FeCl}_{3}\right)$ 可能与底物 作用，首先通过单电子转移(SET)生成自由基中间体， 进而再次单电子转移至 $\mathrm{FeCl}_{3}$ 生成正离子中间体, 因此 该反应需要在 2 equiv. 以上的 $\mathrm{FeCl}_{3}$ 存在下方可进行.

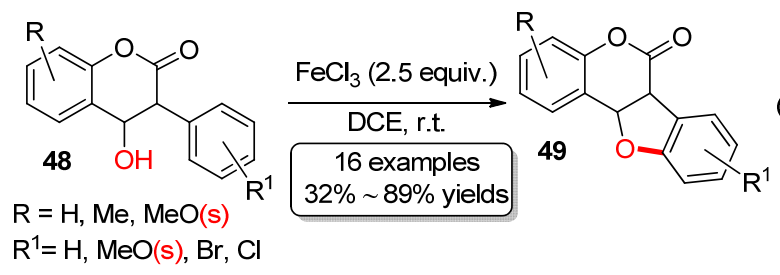

\section{2 芳香烃和苯酚脱氢偶联}

酚羟基较易被氧化，因此苯酚衍生物作为偶联试剂 的报道相对较少，且多数反应为分子内偶联. 2011 年, 刘䂞课题组 ${ }^{[31]}$ 报道了 $\mathrm{Pd}$ 催化的 2-羟基联苯的分子内偶 联反应，实现了二芳基醚结构二苯并呋喃(Eq. 17)的合 成. 酚羟基在反应中既作为导向基团，促进其邻位 $\mathrm{C}-$ $\mathrm{H}$ 键的活化; 同时本身作为偶联组分, 用于构建新的 $\mathrm{C}$ - $\mathrm{O}$ 键. 作者将 2-差基联苯与等物质的量的的 $\mathrm{Pd}$ 催化 剂和卡宾配体混合得到环钯化合物 52, 该化合物在 $120{ }^{\circ} \mathrm{C}$ 下以 $58 \%$ 的产率转化为二氢苯并呋喃. 同位素标 记实验发现 $\mathrm{C}-\mathrm{H}$ 键和 $\mathrm{C}-\mathrm{D}$ 键的活化得到相同的产率. 因此，作者认为该反应的决速步骤是二价钯中间体 $\mathrm{C}-$ $\mathrm{O}$ 键的还原消除步骤，反应经历 $\mathrm{Pd}(0) / \mathrm{Pd}(\mathrm{II})$ 催化循环.
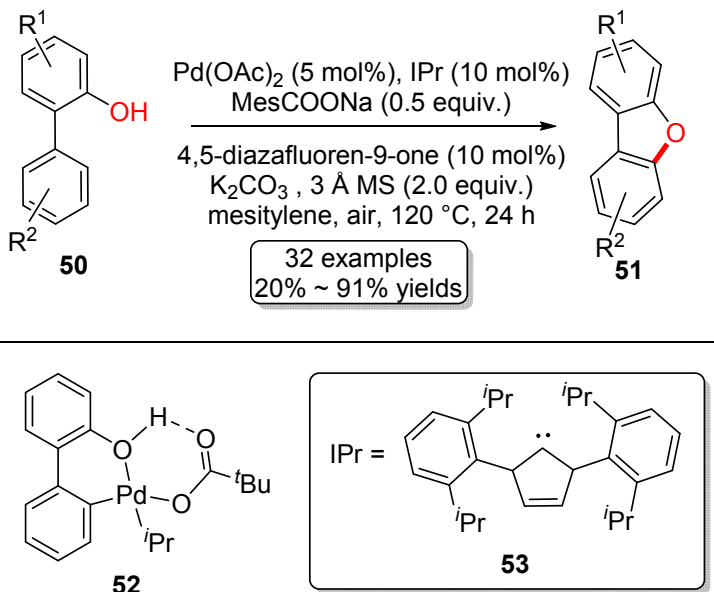

同年, Yoshikai 课题组 ${ }^{[32]}$ 也报道了 2-羟基联苯的分 子内偶联反应(Eq. 18). 反应以 3-硝基吡啶作为配体，过 氧化苯甲酸叔丁酯(tert-butyl peroxybenzoate, $\mathrm{BzOO}^{t} \mathrm{Bu}$ ) 作为氧化剂. 不同于刘否教授发展的反应体系，作者通 过动力学同位素效应(KIE)发现在该条件下, 反应经历 $\mathrm{Pd}(\mathrm{II}) / \mathrm{Pd}(\mathrm{IV})$ 催化循环, 而 $\mathrm{C}-\mathrm{H}$ 键断裂则为反应的决 速步骤。 

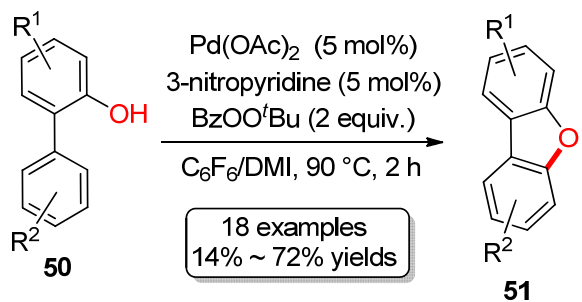

2012 年, 朱强课题组 ${ }^{[33]}$ 发展了铜催化活化 $\mathrm{C}\left(\mathrm{sp}^{2}\right)$ $\mathrm{H}$ 键构建 $\mathrm{C}-\mathrm{O}$ 键的策略(Eq. 19), 该方法可快速制备二 苯并呋喃衍生物. 该反应成功的关键在于酚羟基对位强 吸电子取代基的存在, 可能的原因是强吸电子基团的存 在可防止苯酚结构的氧化. 作者推测了两种可能的反应 机理: (I)铜催化剂通过协同金属去质子化(CMD)的过程 活化邻位 $\mathrm{C}-\mathrm{H}$ 键生成环金属化合物 $\mathbf{5 4}$, 还原消除得到 二苯并呋喃衍生物; (II)铜催化剂与芳环作用生成 $\eta^{2}$ 络 合物 55, 酚羟基亲核进攻铜络合的双键生成 56,56 经过 顺式 $\beta$-H 消除得到二苯并呋喃(Scheme 9).

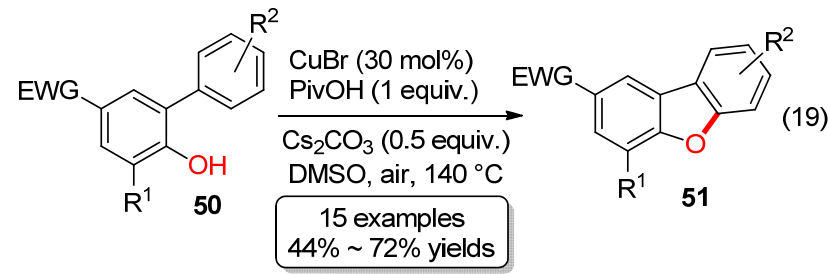

$\mathrm{EWG}=\mathrm{NO}_{2}, \mathrm{CN}, \mathrm{CHO}$;

$\mathrm{R}^{1}=\mathrm{H}, \mathrm{F}, \mathrm{I}, \mathrm{Ph} ; \mathrm{R}^{2}=\mathrm{H}, \mathrm{Me}, \mathrm{OMe}, \mathrm{F}, \mathrm{Cl}, \mathrm{Br}, \mathrm{Ph}$

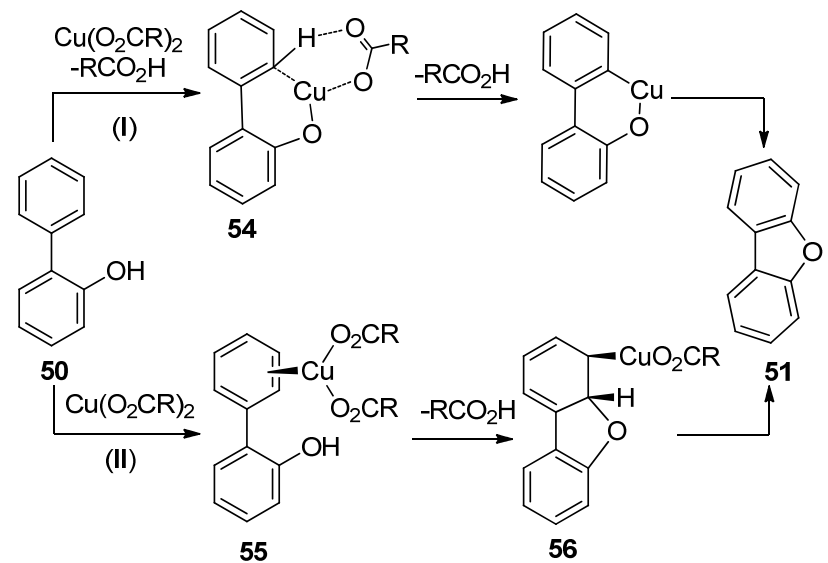

图式 9 铜催化分子内 $\mathrm{C}-\mathrm{H}$ 芳氧基化机理图

Scheme 9 Mechanism for $\mathrm{Cu}$-catalyzed intramolecular aryloxylation.

同年, 该课题组 ${ }^{[34]}$ 研究了含酰胺导向基团的邻芳 基苯酚的分子内 $\mathrm{C}-\mathrm{H}$ 醚化反应(Eq. 20). 该反应中, 酰 胺导向基团不仅控制反应的区域选择性，同时也增加了 反应的活性. 另外, 当过量碘化亚铜 $(\mathrm{CuI})$ 存在时, 苯酚 环首先发生碘代反应, 然后铜催化分子内 $\mathrm{C}-\mathrm{H}$ 醚化反 应环生成碘代二苯并呋喃(Eq. 21).<smiles>[R]C(=O)Nc1cccc(-c2cc([N+](=O)[O-])ccc2O)c1NC([R])=O</smiles><smiles></smiles>
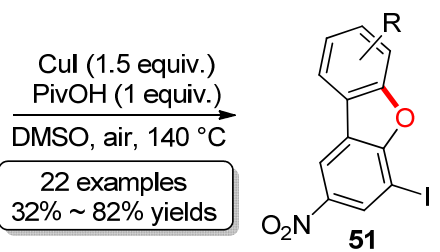

在苯甲酸衍生物的 $\mathrm{C}-\mathrm{H}$ 烷氧基化反应中(Eq. 22), Daugulis 等 ${ }^{[35]}$ 发现当反应的溶剂为 $N, N$-二甲基甲酰胺 (DMF), 碱为碳酸钾 $\left(\mathrm{K}_{2} \mathrm{CO}_{3}\right)$ 时, 8 -氨基喹啉保护的苯甲 酸衍生物可与苯酚衍生物偶联, 实现其邻位 $\mathrm{C}-\mathrm{H}$ 键的 苯氧基化, 生成二芳基醚产物. 酯基、胺基、硝基、腈 基和卤素等官能团取代的苯甲酸衍生物均能顺利进行 反应, 高效得到目标产物. 2014 年, 宋毛平课题组 ${ }^{[36]}$ 利 用 2-氨基吡啶 $-N$-氧化物双齿导向基团, 实现了铜介导 的邻位 $\mathrm{C}-\mathrm{H}$ 苯氧基化反应(Eq. 23).<smiles>[R]#Cc1ccccc1C(=O)Nc1cccc2cccnc12</smiles>

59

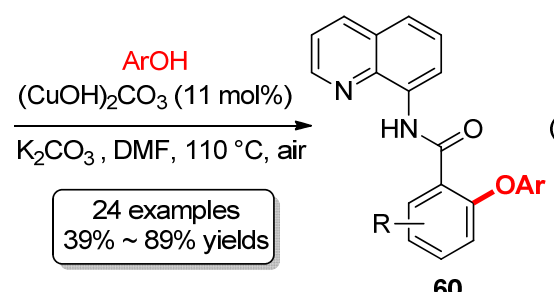

60

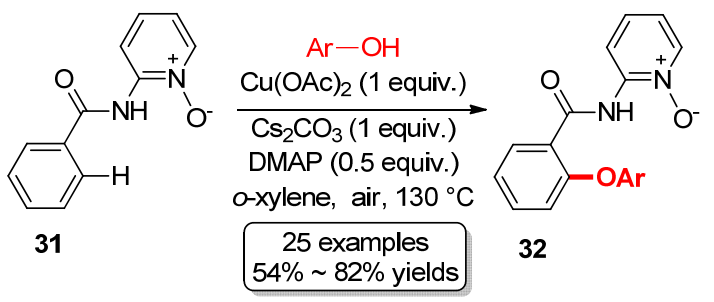

2016 年, 朱维良课题组 ${ }^{[37]}$ 报道了一种二茶氧氮杂 草酮的合成方法(Scheme 10). 首先是 8-氨基喹啉保护的 羧酸衍生物与邻溴苯酚偶联生成二芳基醚化合物 $\mathbf{6 3}$, 卤代芳环进而发生胺化反应生成二苯氧氮杂草酮衍生 物. 作者认为芳基醚化合物 $\mathbf{6 3}$ 通过 Goldberg 胺化生成 非重排产物, 而在碱存在发生 Smile 重排从而胺化生成 重排产物。

\section{$3 \mathrm{C}\left(\mathrm{sp}^{3}\right)$ - $\mathrm{H}$ 键苯氧基化制备芳基醚}

与 $\mathrm{C}\left(\mathrm{sp}^{2}\right)-\mathrm{H}$ 键和醇或酚偶联反应相比, 通过活化 


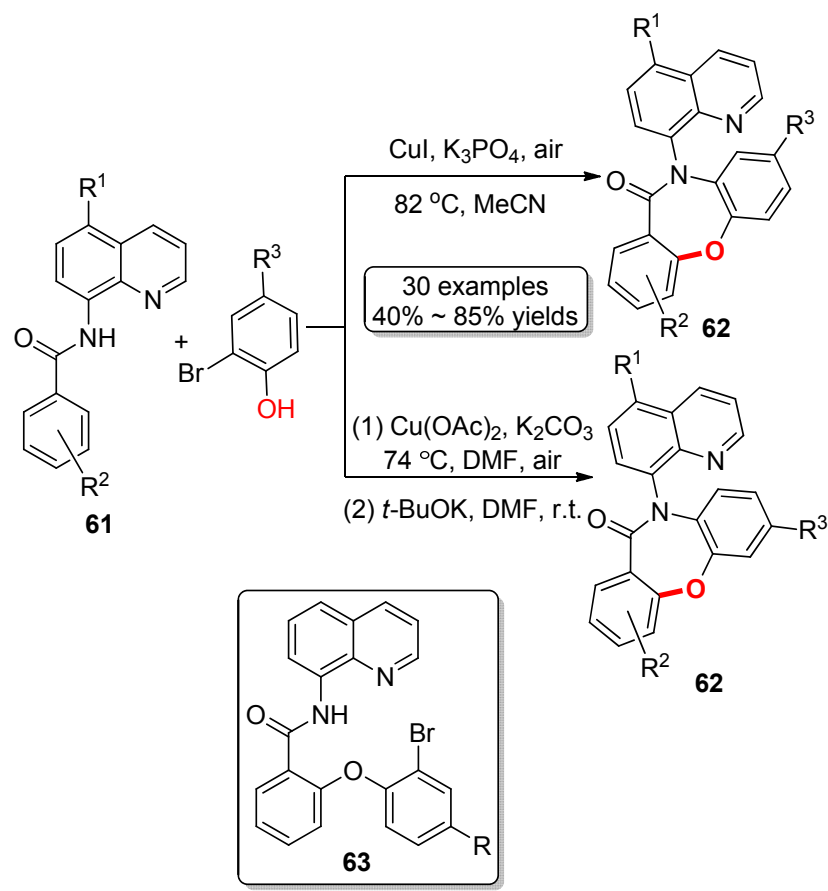

图式 10 苯二氮草类化合物的合成

Scheme 10 Synthesis for the benzodiazepine derivative

$\mathrm{C}\left(\mathrm{sp}^{3}\right)-\mathrm{H}$ 键制备芳香醚的方法报道仍然较少. Kappe 等 ${ }^{[38]}$ 报道了一种醚 $\alpha$-位的 $\mathrm{C}-\mathrm{H}$ 苯氧基化反应(Eq. 24). 反应以叔丁基过氧化氢(tert-butyl hydroperoxide, TBHP) 作为氧化剂, 在铜的催化下进行. 该反应中的苯酚底物 受限于酚羟基邻位含羰基的化合物, 可能的原因是铜催 化剂需要与酚羟基氧原子以及羰基氧原子形成双配位 的中间体才能促进反应进行. 2015 年, Chang 和 Wang 等 ${ }^{[39]}$ 在类似的条件下, 实现了对氧化剂敏感的水杨醛 与醚的偶联反应(Eq. 25). 另外该反应还可以在铁的催 化下进行 ${ }^{[40]}$.
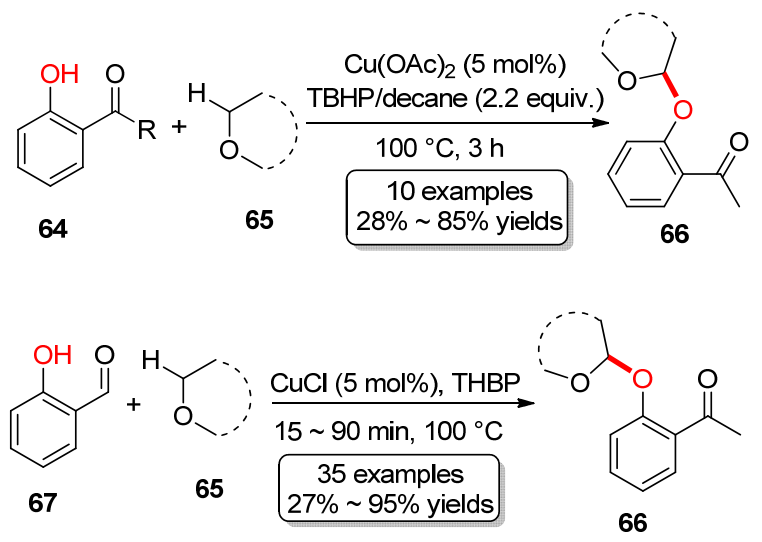

2014 年, Wang 课题组 ${ }^{[41]}$ 报道了四正丁基碘化铵 (TBAI)催化的分子内 $\mathrm{C}\left(\mathrm{sp}^{3}\right)-\mathrm{H}$ 键苯氧基化反应, 实现 了 $\alpha$-酮苯并啞唑衍生物的合成(Eq. 26). 反应在室温下 即可进行, 且具有较好的官能团耐受性. 其可能的机理
如 Scheme 11 所示: 催化剂 TBAI 与氧化剂 TBHP 作用 生成次碘酸盐 70. 该中间体与烯醇 71 作用生成中间体 72. 酚羟基分子内亲核进攻得到环化产物 73, 并重新生 成催化剂 TBAI, 最终 73 在碱性条件下脱磺酰化得到 $\alpha$ 酮苯并啞唑.
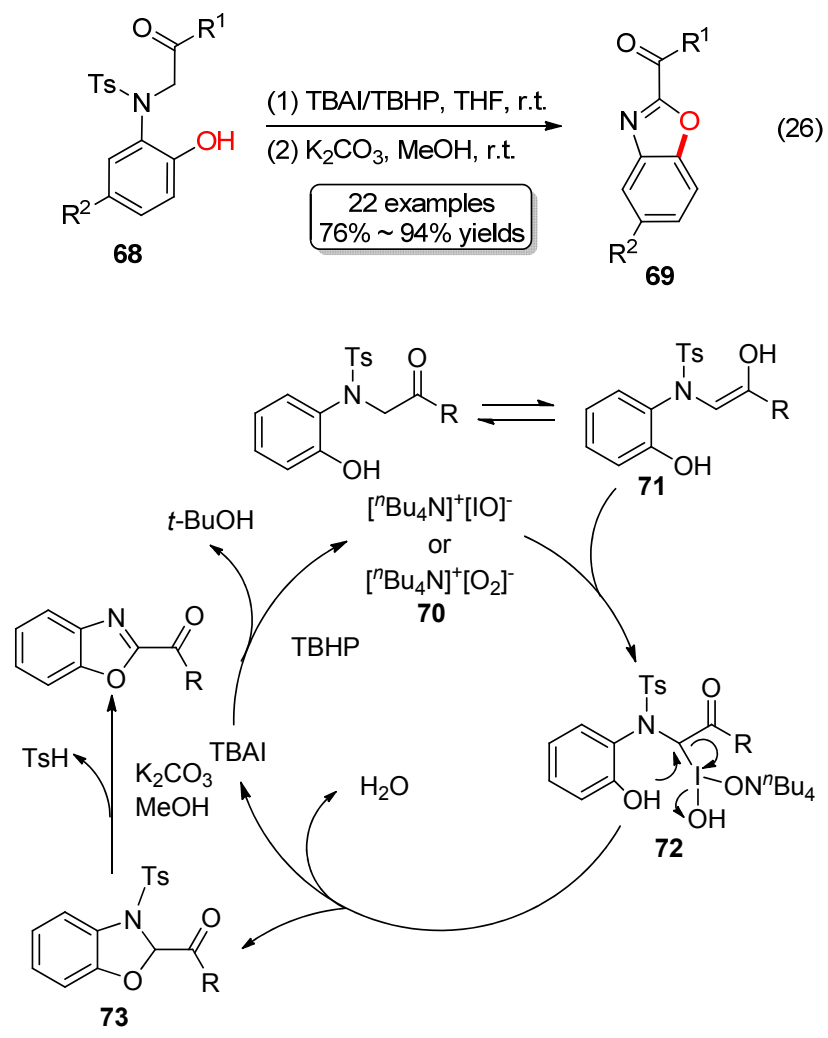

图式 11 四丁基碘化铵催化下的芳烃分子内脱氢机理图 Scheme 11 Ammonium tetrabutyl iodide catalyst dehydrogenative aryl intramolecular mechanism

\section{4 总结与展望}

近年来基于脱氢偶联策略制备芳香醚衍生物的方 法已经取得了快速发展. 目前, 芳香烃与醇的脱氢偶联 研究相对较为成熟, 其主要在 $\mathrm{Pd}$ 或 $\mathrm{Cu}$ 催化下进行. 但 是此类方法一般需要用醇作为反应溶剂，这对芳烃与复 杂醇的反应造成了较大限制. 与此同时，也有不少文献 报道了芳香烃与酚偶联制备二芳基醚化合物的方法，但 是反应往往是在分子内发生，而分子间的芳香烃与苯酚 偶联的方法报道仍然较少. 利用 $\mathrm{C}\left(\mathrm{sp}^{3}\right)-\mathrm{H}$ 键的活化制 备芳基烷基醚的方法亦有报道，但是方法单一，并且具 有较大的局限性.

综上所述，尽管芳香烃与醇或酚之间的脱氢偶联为 芳香醚的制备提供了绿色经济的途径，但是已报道的方 法大多数需要额外的氧化剂, 真正以氢气为唯一副产物 的方法极少. 因此, 设计新型的氧化还原中性的催化剂 体系, 发展条件温和、普适性高的 $\mathrm{C}-\mathrm{H}$ 烷氧基化或苯 
氧基化反应来制备芳基醚化合物，仍是该领域研究的长 期目标. 另外，通过丰富反应底物类型，使相关策略能 应用于复杂的天然产物和药物分子的合成中, 这无疑具 有极其重要的学术和应用价值.

\section{References}

[1] (a) Jabran, K.; Ehsanullah; Hussain, M.; Farooq, M.; Babar, M.; Doğan M.-N.; Lee, D.-J. Weed Biol. Manage. 2012, 12, 136.

(b) Negro, R.; Formoso, G.; Mangieri, T.; Pezzarossa, A.; Dazzi, D.; Hassan, H. J. Clin. Endocrinol. Metab. 2006, 91, 2587.

(c) Kosenkova, Y.-S.; Polovinka, M.; Komarova, N.; Korchagina, D.; Kurochkina, N. Y.; Cheremushkina, V.; Salakhutdinov, N. Chem. Nat. Compd. 2007, 43, 712 .

(d) Deng, H.; Jung, J.-K.; Liu, T.; Kuntz, K. W.; Snapper, M. L.; Hoveyda, A. H. J. Am. Chem. Soc. 2003, 125, 9032.

[2] (a) Chen, G.; Du, J. Chin. J. Org. Chem. 2014, 34, 65 (in Chinese). (陈国军, 杜建时, 有机化学, 2014, 34, 65.)

(b) Tobisu, M.; Chatani, N. Acc. Chem. Res. 2015, 48, 1717.

[3] (a) Ullmann, F.; Sponagel, P. Ber. Dtsch. Chem. Ges. 1905, 38, 2211.

(b) Xiao, S.; Zhu, J.; Mu, X.; Li, Z. Chin. J. Org. Chem. 2013, 33, 1668 (in Chinese).

(肖尚友, 朱俊, 穆小静, 李正华, 有机化学, 2013, 33, 1668.)

[4] (a) Chan, D. M. T.; Monaco, K. L.; Wang, R.-P.; Winters, M. P. Tetrahedron Lett. 1998, 39, 2933.

(b) Evans, D. A.; Katz, J. L.; West, T. R. Tetrahedron Lett. 1998, 39, 2937.

(c) Lam, P. Y. S.; Vincent, G.; Clark, C. G.; Deudon, S.; Jadhav, P. K. Tetrahedron Lett. 2001, 42, 3415 .

[5] (a) Aranyos, A.; Old, D. W.; Kiyomori, A.; Wolfe, J. P.; Sadighi, J. P.; Buchwald, S. L. J. Am. Chem. Soc. 1999, 121, 4369.

(b) Torraca, K. E.; Huang, X.; Parrish, C. A.; Buchwald, S. L. J. Am. Chem. Soc. 2001, 123, 10770.

(c) Mann, G.; Hartwig, J. F. J. Am. Chem. Soc. 1996, $118,13109$.

(b) Mann, G.; Incarvito, C.; Rheingold, A. L.; Hartwig, J. F. J. Am. Chem. Soc. 1999, 121, 3224.

(c) Hartwig, J. F. Nature 2008, 455, 314

[6] Terrett, J. A.; Cuthbertson, J. D.; Shurtleff, V. W.; MacMillan, D. W. C. Nature 2015, 524, 330

[7] (a) Yang, F.; Zhang, H.; Liu, X.; Wang, B.; Ackermann, L. Chin. J. Org. Chem. 2019, 39, 59 (in Chinese)

(杨帆致, 张晗, 刘旭日, 王博, Lutz A., 有机化学, 2019, 39, 59.)

(b) Leila, R.; Asieh, Y.; Mehdi, S. ChemCatChem 2018, 10, 20.

(c) Kamellia, N.; Sheida, A.; Mohammad, N.; Parvaneh, D. K. N.; Esmail, V. RSC Adv. 2018, 8, 19125 .

(d) Bhunia, S.; Pawar, G. G.; Kumar, S. V.; Jiang, Y.; Ma, D. Angew. Chem., Int. Ed. 2017, 56, 16136.

(e) Ding, H.; Li, J.; Guo, Q.; Xiao, Y. Chin. J. Org. Chem. 2017, 37, 3112 (in Chinese)

(丁怀伟, 李娟, 郭庆辉, 肖琰, 有机化学, 2017, 37, 3112.)

[8] Desai, L. V.; Malik, H. A.; Sanford, M. S. Org. Lett. 2006, 8, 1141.

[9] Wang, G.-W.; Yuan, T.-T. J. Org. Chem. 2010, 75, 476.

[10] Jiang, T.-S.; Wang, G.-W. J. Org. Chem. 2012, 77, 9504

[11] Shi, S.-P.; Kuang, C.-X. J. Org. Chem. 2014, 79, 6105.

[12] Li, W.; Sun, P.-P. J. Org. Chem. 2012, 77, 8362

[13] Yin, Z.-W.; Jiang, X.-Q.; Sun, P.-P. J. Org. Chem. 2013, 78, 10002
[14] Zhang, C.; Sun, P.-P. J. Org. Chem. 2014, 79, 8457.

[15] Gao, T.-T.; Sun, P.-P. J. Org. Chem. 2014, 79, 9888.

[16] Peron, F.; Fossey, C.; Santos, J. O. S.; Cailly, T.; Fabis, F. Chem.Eur. J. 2014, 20, 1

[17] Chen, F.-J.; Zhao, S.; Hu, F.; Chen, K.; Zhang, Q.; Zhang, S.-Q.; Shi, B.-F. Chem. Sci. 2013, 4, 4187.

[18] (a) Shen, T.; Wang, X.-N.; Lou, H.-X. Nat. Prod. Rep. 2009, 26, 916.

(b) Veitch, N. C. Nat. Prod. Rep. 2007, 24, 417.

(c) Watzke, A.; O'Malley, S. J.; Bergman, R. G.; Ellman, J. A. J. Nat. Prod. 2006, 69, 1231.

(d) Tsui, G. C.; Tsoung, J.; Dougan, P.; Lautens, M. Org. Lett. 2012, 14,5542 .

[19] Wang, X.-S.; Lu, Y.; Dai, H.-X.; Yu, J.-Q. J. Am. Chem. Soc. 2010 , $132,12203$.

[20] Wang, H.-B.; Li, G.; Engle, K. M.; Yu, J.-Q.; Davies, H. M. L. J. Am. Chem. Soc. 2013, 135, 6774.

[21] Cheng, X.-F.; Li, Y.; Su, Y.-M.; Yin, F.; Wang, J.-Y.; Sheng, J.; Vora, H. U.; Wang, X.-S.; Yu, J.-Q. J. Am. Chem. Soc. 2013, 135, 1236.

[22] Wang, Z.-L.; Zhao, L.; Wang, M.-X. Org. Lett. 2011, 13, 6560.

[23] Zhang, L.-B.; Hao, X.-Q.; Zhang, S.-K.; Liu, K.; Ren, B.-Z.; Gong, J.-F.; Niu, J.-L.; Song, M.-P. J. Org. Chem. 2014, 79, 10399.

[24] Bhadra, S.; Matheis, C.; Katayev, D.; Gooßen, L. J. Angew. Chem. 2013, 125, 9449 .

[25] Roane, J.; Daugulis, O. Org. Lett. 2013, 15, 5842.

[26] Yin, X.-S.; Li, Y.-C.; Yuan, J.; Gua, W.-J.; Shi, B.-F. Org. Chem. Front. 2015, 2, 119.

[27] Zhang, L.-B.; Hao, X.-Q.; Zhang, S.-K.; Liu, Z.-J.; Zheng, X.-X.; Gong, J.-F.; Niu, J.-F.; Song, M.-P. Angew. Chem., Int. Ed. 2015, 54,272

[28] Zheng, Y.-W.; Ye, P.; Chen, B.; Meng, Q.-Y.; Feng, K.; Wang, W.-G.; Wu, L.-Z.; Tung, C.-H. Org. Lett. 2017, 19, 2206.

[29] Ohkubo, K.; Kobayashi, T.; Fukuzumi, S. Opt. Express 2012, 20, A360.

[30] Tang, L.; Pang, Y.; Yan, Q.; Shi, L.-Q.; Huang, J.-H.; Du, Y.-F.; Zhao, K. J. Org. Chem. 2011, 76, 2744.

[31] Xiao, B.; Gong, T.-J.; Liu, Z.-J.; Liu, J.-H.; Luo, D.-F.; Xu, J.; Liu L. J. Am. Chem. Soc. 2011, 133, 9250.

[32] Wei, Y.; Yoshikai, N. J. Org. Chem. 2011, 13, 5504.

[33] Zhao, J.-J.; Wang, Y.; He, Y.-M.; Liu, L.-Y.; Zhu, Q. Org. Lett. 2012, 14, 1078.

[34] (a) Zhao, J.-J.; Wang, Y.; Zhu, Q. Synthesis 2012, 44, 1551. (b) Zhao, J.-J.; Zhang, Q.; Liu, L.-Y.; He, Y.-M.; Li, J.; Li, J.; Zhu, Q. Org. Lett. 2012, 14, 5362.

[35] Roane, J.; Daugulis, O. Org. Lett. 2013, 15, 5842.

[36] Hao, X.-Q.; Chen, L.-J.; Ren, B.; Li, L.-Y.; Yang, X.-Y.; Gong, J.-F.; Niu, J.-L.; Song, M.-P. Org. Lett. 2014, 16, 1104.

[37] Zhou, Y.-F.; Zhu, J.-M.; Li, B.; Zhang, Y.; Feng, J.; Hall, A.; Shi, J.-Y.; Zhu, W.-L. Org. Lett. 2016, 18, 3803

[38] Kumar, G. S.; Pieber, B.; Reddy, K. R.; Kappe, C. O. Chem.-Eur. J. 2012, 18, 6124.

[39] Barve, B. D.; Wu, Y.-C.; El-Shazly, M.; Korinek, M.; Cheng, Y.-B.; Wang, J.-J.; Chang, F.-R. Tetrahedron 2015, 71, 2290

[40] Barve, B. D.; Wu, Y.-C.; El-Shazly, M.; Korinek, M.; Cheng, Y.-B.; Wang, J.-J.; Chang, F.-R. Org. Lett. 2014, 16, 1912.

[41] Boominathan, S. S. K.; Hu, W.-P.; Senadi, G. C.; Vandavasia, J. K.; Wanga, J.-J. Chem. Commun. 2014, 50, 6726. 